\title{
A LOCALLY COMPACT NONPARACOMPACT SPACE FOR WHICH THE STRICT TOPOLOGY IS MACKEY
}

\author{
ROBERT F. WHEELER
}

ABSTRACT. A space as described in the title is used to construct counterexamples to several conjectures in the theory of locally convex spaces.

The purpose of this note is to show that a certain locally compact Hausdorff space $X$, originally conceived and examined by Negrepontis [10], has interesting properties from the point of view of functional analysis and topological measure theory. In particular, the space $\left(C^{*}(X), \beta\right)$ of realvalued bounded continuous functions on $X$, endowed with the strict topology, has the property described by the title. Moreover, counterexamples to several recent conjectures concerning completeness of locally convex spaces can be readily obtained. Finally, the existence of $X$ is used to show that a variant of semireflexivity for locally convex spaces, introduced by the author in [14], is indeed a distinct (properly stronger) concept.

1. Topological properties of the space $X$. The basic construction is sketched here; see [10] for more details. The continuum hypothesis is assumed throughout. Let $p$ be a $P$-point of $\beta N \backslash N$, and let $\left\{G_{\alpha}\right\}_{\alpha<\omega_{1}}$ be a base of clopen neighborhoods of $p$ in $\beta N \backslash N$, such that $G_{\alpha} \varsubsetneqq G_{\beta}$ for $\beta<\alpha$. Define inductively a family of nonempty pairwise disjoint clopen sets $\left\{V_{a}\right\}_{a<\omega_{1}}$ in $\beta N \backslash N$ such that $V_{a} \subset G_{a} \backslash G_{a+1}$ for all $a<\omega_{1}$. Let $E=$ $\cup_{a<\omega_{1}} V_{a}$, and let $X=N \cup E$ (with the relative topology of $\beta N$ ). Then $X$ is locally compact, $\beta X=\beta N$, and the Hewitt realcompactification of $X$ is given by $\nu X=X \cup\{p\}[10]$; moreover, $\nu X$ is Lindelöf. It follows from Shirota's theorem $[6, \mathrm{p} .229]$ that $\nu X=\theta X$; here $\theta X$ denotes the topological space underlying the completion of $X$ with the fine uniformity (see [1]). Negrepontis goes on to construct a quotient space of $X$, but, for simplicity, we shall consider only $X$ itself.

Presented to the Society, April 27, 1973; received by the editors April 30, 1974. AMS (MOS) subject classifications (1970). Primary 46E10, 54C35; Secondary 54D45, 54D60.

Key words and phrases. $\mu$-space, P-point, strict topology, strong Mackey space. 
We shall require one additional topological result. A subset $B$ of a completely regular Hausdorff space $T$ is bounded (older terminology: relatively pseudocompact) if the restriction to $B$ of any real-valued continuous function on $T$ is bounded. Then $T$ is a $\mu$-space [1] if every bounded subset of $T$ is relatively compact. Any topologically complete space is a $\mu$-space; the converse fails, but the only published counterexamples known to the author $([6,9 \mathrm{~L}]$ and $[8])$ are not locally compact. Note that the classical Nachbin-Shirota result may be phrased: $(C(T)$, co-op) is a barrelled locally convex space if and only if $T$ is a $\mu$-space.

Lemma 1. The space $X$ is a $\mu$-space.

Proof. Let $B$ be a bounded subset of $X$. We show first that $B$ meets only finitely many $V_{a}$. If not, there is an increasing sequence $a_{1}<\alpha_{2}<\cdots$ of countable ordinals and a sequence $\left(x_{n}\right)$ in $X$ with $x_{n} \in V_{a_{n}} \cap B \forall n$. Let $\alpha_{0}=\sup \alpha_{n}<\omega_{1}$, and choose a subset $M_{1}$ of $N$ with $\operatorname{cl}_{\beta N} M_{1}=M_{1} \cup$ $\left((\beta N \backslash N) \backslash G_{a_{0}}\right)[6,6 S]$. Then $Y=X \cap \mathrm{cl}_{\beta N} M_{1}$ is $\sigma$-compact (hence a $\mu$ space), and $B \cap Y$ is a bounded subset of $Y$, but $\left(x_{n}\right)$ has no cluster point in $Y$, a contradiction.

To complete the proof, we may assume that $B$ is closed in $X$. Let

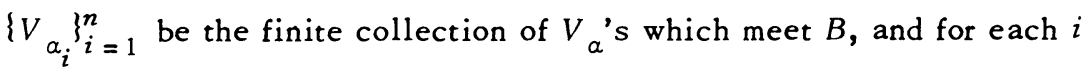
choose $N_{i} \subset N$ with cl $\beta N N_{i}=N_{i} \cup V_{a_{i}}$. Let $M=N \backslash \bigcup_{i=1}^{n} N_{i}$. Since cl $\beta N^{M}$ meets no $V_{a_{i}}, B \cap M$ is bounded, clopen, and discrete in $X$, hence must be finite. It follows that $B$ is compact.

Lemma 2. The point $p$ is an isolated point of every compact subset $K$ of $\theta X$ which contains it.

Proof. Since every continuous real-valued function on $X$ admits a continuous extension to $\theta X, K \cap X$ is a bounded (and closed) subset of $X$. Hence it is compact and therefore closed in $\theta X$.

2. Properties of $\left(C^{*}(X), \beta\right)$. A locally convex space $E$ is said to be a strong Mackey space if every relatively weak ${ }^{*}$-countably compact ${ }^{1}$ subset of the dual space $E^{\prime}$ is equicontinuous. If $S$ is paracompact locally compact, a well-known result of Conway [3] states that $\left(C^{*}(S), \beta\right)$ is a strong Mackey space, where $\beta$ is the strict topology introduced by Buck [2]. The space $X$

${ }^{1}$ A subset $H$ of $E^{\prime}$ is relatively weak ${ }^{*}$-countably compact if everv sem...... in $H$ has a weak ${ }^{*}$-cluster point in $E^{\prime}$. 
described here yields the first locally compact nonparacompact example of the strong Mackey property known to the author. We find it convenient to examine $C^{*}(\theta X)$ initially and then apply the results to $C^{*}(X)$ itself. Since $\theta X$ is not locally compact (as Lemma 2 shows), it is appropriate to consider the strict topologies $\beta_{0}, \beta$, and $\beta_{1}$ on $C^{*}(T)$ (T completely regular) introduced by Sentilles [11]. Recall in particular that these topologies yield the spaces $M_{t}(T), M_{\tau}(T)$, and $M_{\sigma}(T)$ of Varadarajan [13] as dual spaces of $C^{*}(T)$, and that $\beta_{0}=\beta$ is the usual strict topology for locally compact $T$.

Proposition 1. $\left(C^{*}(\theta X), \beta_{0}\right)$ is a strong Mackey space.

Proof. It is enough to show that $\beta_{0}=\beta=\beta_{1}[11$, Theorem 4.5]. Since $\theta X$ is Lindelöf and a Borel set in its Stone-Čech compactification, we have $M_{\sigma}(\theta X)=M_{\tau}(\theta X)=M_{t}(\theta X)$ [13]. According to Theorem 2.13 of [9], it now suffices to show that $\theta X$ is a $T$-space (every weak ${ }^{*}$-compact subset $H$ of $M_{t}^{+}(\theta X)$ is uniformly tight. $)^{2}$ The idea is to split $\theta X$ into three parts, corresponding to the following known examples of $T$-spaces: (1) P-spaces [15]; (2) locally compact spaces; and (3) spaces $N \cup\{q\}$, where $q \in \beta N \backslash N[13$, p. 227]. ${ }^{3}$

Now $W=E \cup\{p\}$ is closed in $\theta X$, so $H \mid W=\{\mu \mid W: \mu \in H\}$ is relatively weak ${ }^{*}$-compact in $M_{t}^{+}(W)$ [9, Theorem 4.5]. Now the space obtained from $W$ by shrinking each $V_{a}$ to a point is a $P$-space, and it follows from (1) and Theorem 4.3 of [9] that $H \mid W$ is uniformly tight. The characterization of compact sets in Lemma 1 shows that, for some $a_{0}<\omega_{1},|\mu|\left(U_{a \geq \alpha_{0}} V_{\alpha}\right)=0$ $\forall \mu \in H$. Choose $M \subset N$ with $\mathrm{cl}_{\beta N} M=M \cup G_{a_{0}}$, and let $Y=\mathrm{cl}_{\beta N} M \cap \theta X$. Since $Y$ is clopen in $\theta X, H \mid Y$ is $\sigma\left(M_{t}(Y), C^{*}(Y)\right)$-compact. Now each $\mu$ in $H$, when restricted to the Borel sets of $Y$, satisfies $|\mu|(Y \backslash D)=0$, where $D=M \cup\{p\}$. Since $D \subset Y \subset \operatorname{cl}_{\beta N} D=\beta D$, it is not hard to show that $H \mid D$ is $\sigma\left(M_{t}(D), C^{*}(D)\right)$-compact. Hence, from (3), $H \mid D$ (and therefore $H \mid Y$ ) is uniformly tight. Now it follows from (2) that $H \mid \theta X \backslash Y$ is uniformly tight as well, and this establishes the result.

Corollary. $\left(C^{*}(X), \beta\right)$ is a strong Mackey space.

Proof. Let $H \subset M_{t}(X)$ be relatively weak ${ }^{*}$-countably compact. The embedding $j: X \rightarrow \theta X$ induces a weak ${ }^{*}$-continuous map $j^{*}: M_{t}(X) \rightarrow M_{t}(\theta X)$.

$2 T$-spaces are referred to by many workers as Prohorov spaces.

3 A strong result containing both (1) and (3) has been obtained recently by Haydon [7]. 
Then $j^{*}(H)$ is uniformly tight, from Proposition 1 , and it follows from Lemma 2 that $H$ is uniformly tight. Thus $\left(C^{*}(X), \beta_{0}\right)$ is strong Mackey, but $\beta_{0}=\beta$ since $X$ is locally compact.

Since $X$ admits no complete uniform structure (being a proper subset of $\theta X)$, it is not paracompact, ${ }^{4}$ nor does it satisfy the measure-theoretic property $M_{\tau}(X)=M_{S}(X)$ introduced in [16] as a sufficient condition for the Mackey property. Moreover, the Banach algebra $C_{0}(X)$ has no well-behaved approximate identity in the sense of Taylor [12]. Thus it appears that the elusive necessary and sufficient condition for the Mackey property has not been achieved by present theories.

3. Some applications to completeness of locally convex spaces. In a recent survey address, Buchwalter [1] posed a question which we rephrase as: If $(E, \mathfrak{J})$ is a complete locally convex space, and $\mathcal{J}_{K}$ is the finest locally convex topology on $E$ which agrees with $\mathcal{T}$ on $\mathcal{T}$-compact absolutely convex sets, is $\left(E, \mathcal{T}_{K}\right.$ ) again complete? A similar problem (delete "absolutely convex") was raised by Frölicher and Jarchow [5]. Finally, Dazord and Jourlin [4] have considered the problem: Is a locally convex space in which closed totally bounded sets are compact necessarily quasi-complete?

All three questions are resolved negatively by an example of Haydon [8]. By an ingenious construction he obtains a (nonlocally compact) space $T$ such that $(C(T)$, co-op) is complete, but $(C(\theta T)$, co-op) is not quasicomplete, although closed totally bounded sets in the latter space are compact.

The space $X$ described in this note furnishes a strict topology analogue of Haydon's example. Let $E=C^{*}(X)$ and $\mathfrak{T}=\beta$. Then $\mathfrak{J}_{K}$ is the topology $\beta_{e}$ described in [16]. There is a natural vector space isomorphism between $C^{*}(X)$ and $C^{*}(\theta X)$; because $X$ and $\theta X$ have the same continuous metric images [17, Theorem 2.4], this correspondence is a topological isomorphism between $\left(C^{*}(X), \beta_{e}\right)$ and $\left(C^{*}(\theta X), \beta_{e}\right)$. Since $\beta_{0}=\beta=\beta_{e}=\beta_{1}$ for $\theta X$ (Proposition 1), we have an identification between $\left(C^{*}(X), \beta_{e}\right)$ and $\left(C^{*}(\theta X)\right.$, $\left.\beta_{0}\right)$. The former has the property that closed totally bounded sets are compact [16], while the latter is not quasicomplete: the characteristic function of $p$ is continuous on compact subsets of $\theta X$, by Lemma 2, but not continuous. Apply Theorem 7.1 of [11].

We note that $\beta_{e}$ convergence can be described quite precisely here: a

${ }^{4} X$ is not even normal, since it is a separable space with an uncountable closed discrete subset. 
bounded net $\left(f_{a}\right)$ in $C^{*}(X)$ is $\beta_{e}$-convergent to $f$ if and only if (1) $\left(f_{a}\right)$ converges to $f$ in the compact-open topology, and (2) $\bar{f}_{\alpha}(p) \rightarrow \bar{f}(p)$, where the bar denotes the extension to $\theta X$.

Finally, we refer the reader to [14] for a discussion of a type of completeness called "strong semireflexivity." The space $X$ described here satisfies all the conditions enumerated in $\$ 6$ of that paper; hence there is a semireflexive space which is not strongly semireflexive.

\section{REFERENCES}

1. H. Buchwalter, Fonctions continues et mesures sur un espace complètement régulier, Lecture Notes in Math., vol. 331, Springer-Verlag, Berlin and New York, 1973, pp. 183-202.

2. R. C. Buck, Bounded continuous functions on a locally compact space, Michigan Math. J. 5 (1958), 95-104. MR 21 \#4350.

3. J. B. Conway, The strict topology and compactness in the space of measures, Trans. Amer. Math. Soc. 126 (1967), 474-486. MR 34 \#6503.

4. J. Dazord and M. Jourlin, Sur les précompacts d'un espace localement convexe, C. R. Acad. Sci. Paris Sér. A-B 274 (1972), A463-A466, A550-A 553. MR 47 \#751; \#752.

5. A. Frölicher and H. Jarchow, Zür Dualitätstheorie kompakt erzeugter und lokalkonvexer Vektorräume, Comment. Math. Helv. 47 (1972), 289-310.

6. L. Gillman and M. Jerison, Rings of continuous functions, University Ser. in Higher Math., Van Nostrand, Princeton, N. J., 1960. MR 22 \#6994.

7. R. Haydon, On compactness in spaces of measures and measurecompact spaces, Proc. London Math. Soc. 29 (1974), 1-16.

8. - Sur un problème de H. Buchwalter, C. R. Acad. Sci. Paris Sér. A-B 275 (1972), A 1077-A1080. MR 47 \#3931.

9. S. E. Mosiman and R. F. Wheeler, The strict topology in a completely regular setting, Canad. J. Math. 24 (1972), 873-890.

10. S. Negrepontis, An example on realcompactifications, Arch. Math. (Basel) 20 (1969), 162-164. MR 39 \#6265.

11. F. D. Sentilles, Bounded continuous functions on a completely regular space, Trans. Amer. Math. Soc. 168 (1972), 311 -336. MR 45 \#4133.

12. D. C. Taylor, A general Phillips theorem for $C^{*}$-algebras and some applica. tions, Pacific J. Math. 40 (1972), 477-488. MR 46 \#7913.

13. V. S. Varadarajan, Measures on topological spaces, Mat. Sb. 55 (97) (1961), 35-100; English transl., Amer. Math. Soc. Transl. (2) 48 (1965), 161-228. MR 26 \#6342.

14. R. F. Wheeler, The equicontinuous weak ${ }^{*}$-topology and semi-reflexivity, Studia Math. 41 (1972), 243-256. MR 46 \#4150.

15. - The strict topology for P-spaces, Proc. Amer. Math. Soc. 41 (1973), $466-472$.

16. - The strict topology, separable measures, and paracompactness. Pacific J. Math. 47 (1973), 287-302.

17. K. Morita, Topological completions and M-spaces, Sci. Rep. Tokyo Kyoiku Daigaku Sect. A 10 (1970), 271-288. MR $42 \# 6785$.

DEPARTMENT OF MATHEMATICAL SCIENCES, NORTHERN ILLINOIS UNIVERSITY, DE KALB, ILLINOIS 60115 